\title{
The usefulness of SARC-F
}

\author{
Karolina Piotrowicz ${ }^{1}$ Barbara Gryglewska ${ }^{1}$ Jerzy Gąsowski ${ }^{1}$
}

Received: 7 March 2021 / Accepted: 13 March 2021 / Published online: 2 April 2021

(c) The Author(s), under exclusive licence to Springer Nature Switzerland AG 2021

Malas et al. [1] are correct to observe that due to a low sensitivity, the SARC-F test should be used cautiously to screen for sarcopenia. Rather than being used alone to pickup positive cases, thanks to its high specificity which we have mentioned in our manuscript [2], SARC-F may be used to rule out sarcopenia. The patients in whom sarcopenia has not been ruled out should undergo further assessment. Indeed, the diagnosis of sarcopenia should be looked at from a broader perspective, as outlined in the 2018 EWGSOP2 guidelines [3]. After the initial stage, where either clinical suspicion, or the high scoring in SARC-F are used, the assessment involves the hand-grip or chair-stands, which are very much in line with the proposal by Malas et al. Currently, the muscular ultrasound is being assessed within the scope of the SARCUS project, to aid with the diagnosis of sarcopenia [4]. We agree that one of the great challenges of geriatric medicine is to try to prevent sarcopenia rather than fight its consequences. This may be of special importance in the context of the COVID-19, as the disease itself and its aftermath may promote the emergence of acute postCOVID-19 sarcopenia. To find the susceptible patients early, a broader scrutiny of the patient's status should be employed that among other issues would address the possibility of frailty, including the cognitive and social frailty, and the obese sarcopenia. Additionally, as shown by the experience gained during COVID-19, subjective measures such as SARC-F may be administered over the telephone or Internet, and may be easily implemented in the e-health functionalities. The fact that to arrive at diagnoses, subjective measures should be put into a broader context, goes without saying.

\section{Declarations}

Conflict of interest The authors have no conflict of interest in connection with the present work.

Statement of human and animal rights This article does not contain any studies with human participants or animals performed by any of the authors.

Informed consent For this type of study, formal consent is not required.

\section{References}

1. Malas FÜ, Kara M, Özçakar L (2021) SARC-F as a case-finding tool in sarcopenia: valid or unnecessary? Aging Clin Exp Res. https://doi.org/10.1007/s40520-021-01838-7

2. Piotrowicz K, Głuszewska A, Czesak J et al (2021) SARC-F as a case-finding tool for sarcopenia according to the EWGSOP2. National validation and comparison with other diagnostic standards. Aging Clin Exp Res. https://doi.org/10.1007/ s40520-020-01782-y

3. Cruz-Jentoft AJ, Bahat G, Bauer J et al (2019) Sarcopenia: revised European consensus on definition and diagnosis. Age Ageing 48:16-31

4. Perkisas S, Bastijns S, Baudry S et al (2021) Application of ultrasound for muscle assessment in sarcopenia: 2020 SARCUS update. Eur Geriatr Med 12:45-59

Publisher's Note Springer Nature remains neutral with regard to jurisdictional claims in published maps and institutional affiliations.

Jerzy Gąsowski

jerzy.gasowski@uj.edu.pl

1 Department of Internal Medicine and Gerontology, Jagiellonian University Medical College, University Hospital, 2 Jakubowskiego St., building I, 5th floor, 30-688 Kraków, Poland 